\title{
Amplifying the Hawking Signal in BECs
}

\author{
Roberto Balbinot ${ }^{1,2}$ and Alessandro Fabbri, ${ }^{2,3,4}$ \\ ${ }^{1}$ Dipartimento di Fisica dell'Università di Bologna and INFN Sezione di Bologna, Via Irnerio 46, 40126 Bologna, Italy \\ ${ }^{2}$ Museo Storico della Fisica e Centro Studi e Ricerche "Enrico Fermi", Piazza del Viminale 1, 00184 Roma, Italy \\ ${ }^{3}$ Dipartimento di Fisica dell'Università di Bologna, Via Irnerio 46, 40126 Bologna, Italy \\ ${ }^{4}$ Departamento de Física Teòrica and IFIC, Universidad de Valencia-CSIC, C. Dr. Moliner 50, 46100 Burjassot, Spain
}

Correspondence should be addressed to Alessandro Fabbri; afabbri@ific.uv.es

Received 7 January 2014; Accepted 6 March 2014; Published 27 April 2014

Academic Editor: Piero Nicolini

Copyright (c) 2014 R. Balbinot and A. Fabbri. This is an open access article distributed under the Creative Commons Attribution License, which permits unrestricted use, distribution, and reproduction in any medium, provided the original work is properly cited. The publication of this article was funded by $\mathrm{SCOAP}^{3}$.

\begin{abstract}
We consider simple models of Bose-Einstein condensates to study analog pair-creation effects, namely, the Hawking effect from acoustic black holes and the dynamical Casimir effect in rapidly time-dependent backgrounds. We also focus on a proposal by Cornell to amplify the Hawking signal in density-density correlators by reducing the atoms' interactions shortly before measurements are made.
\end{abstract}

\section{Introduction}

Analogue models in condensed matter systems are nowadays an active field of investigation, not only on the theoretical side but, more importantly, also on the experimental one. The underlying idea is to reproduce in a condensed matter context peculiar and interesting quantum effects predicted by Quantum Field Theory in curved space, whose experimental verification in the gravitational context appears at the moment by far out of reach.

Many efforts are devoted to find the most famous of these effects, namely, the thermal emission by black holes predicted by Hawking in 1974 [1]. Among the condensed matter systems under examination, Bose-Einstein condensates appear as the most promising setting to achieve this goal [2-13]. The major problem one has to face experimentally is the correct identification of the signal corresponding to the analogue of Hawking radiation, namely, a thermal emission of phonons as a consequence of a sonic horizon formation, since it can be covered by other competing effects, like large thermal fluctuations.

A major breakthrough to overcome this problem came in 2008, when it was predicted that, as a consequence of Hawking radiation being a genuine pair creation process, a characteristic peak in the density correlation function of the condensate should appear for points situated on opposite sides with respect to the horizon [14]. This is the "smoking gun" of the Hawking effect. Soon after this proposal, Eric Cornell at the first meeting on "experimental Hawking radiation" held in Valencia in 2009 suggested that one can amplify this characteristic signal by reducing the interaction coupling among the atoms of the BEC shortly before measuring the density correlations [15].

Here we review in a simple pedagogical way, using toy models, how the analogous of Hawking radiation occurs in a supersonic flowing BEC and how the corresponding characteristic peak in the correlation function can be amplified according to Cornell's suggestion. It should be stressed that nowadays correlation functions measurements are becoming the basic experimental tool to investigate Hawking-like radiation in condensed matter systems.

\section{BECs: The Gravitational Analogy and Hawking Radiation}

A Bose gas in the dilute gas approximation is described by a field operator $\widehat{\Psi}$ with equal-time commutator (see, e.g., [16])

$$
\left[\widehat{\Psi}(t, \vec{x}), \widehat{\Psi}^{\dagger}\left(t, \vec{x}^{\prime}\right)\right]=\delta^{3}\left(\vec{x}-\vec{x}^{\prime}\right)
$$


satisfying the time-dependent Schrödinger equation

$$
i \hbar \partial_{t} \widehat{\Psi}=\left(-\frac{\hbar^{2}}{2 m} \vec{\nabla}^{2}+V_{\text {ext }}+g \widehat{\Psi}^{\dagger} \widehat{\Psi}\right) \widehat{\Psi}
$$

where $m$ is the mass of the atoms, $V_{\text {ext }}$ is the external potential, and $g$ is the nonlinear atom-atom interaction coupling constant. At sufficiently low temperatures a large fraction of the atoms condense into a common ground state which is described, in the mean field approach, by a $c$-number field $\Psi_{0}(t, \vec{x})$.

To consider linear fluctuations around this classical macroscopic condensate, one writes the bosonic field operator $\widehat{\Psi}$ as

$$
\widehat{\Psi} \sim \Psi_{0}(1+\widehat{\phi})
$$

where $\widehat{\phi}$ is a small (quantum) perturbation. $\Psi_{0}$ and $\widehat{\phi}$ satisfy, respectively, Gross-Pitaevski

$$
i \hbar \partial_{t} \Psi_{0}=\left(-\frac{\hbar^{2}}{2 m} \vec{\nabla}^{2}+V_{\text {ext }}+g n_{0}\right) \Psi_{0}
$$

where $n_{0}=\left|\Psi_{0}\right|^{2}$ is the number density, and Bogoliubov-de Gennes equations

$$
i \hbar \partial_{t} \widehat{\phi}=-\left(\frac{\hbar^{2}}{2 m} \vec{\nabla}^{2}+\frac{\hbar^{2}}{m} \frac{\vec{\nabla} \Psi_{0}}{\Psi_{0}} \vec{\nabla}\right) \hat{\phi}+m c^{2}\left(\widehat{\phi}+\widehat{\phi}^{\dagger}\right)
$$

with $c=\sqrt{g n_{0} / m}$ being the speed of sound.

Contact with the gravitational analogy (see, e.g., [13]) is achieved in the (long wavelength) hydrodynamic approximation, more easily realised by considering the density-phase representation for the Bose operator $\widehat{\Psi}=\sqrt{\widehat{n}} e^{i \widehat{\theta}}$ and the splitting $\widehat{n}=n_{0}+\widehat{n}_{1}, \widehat{\theta}=\theta_{0}+\widehat{\theta}_{1}$ in which $\widehat{n}_{1}, \widehat{\theta}_{1}$ represent the linear (quantum) density and phase fluctuations, respectively. In terms of $\widehat{\phi}$ and $\widehat{\phi}^{\dagger}$ we have

$$
\widehat{n}_{1}=n_{0}\left(\widehat{\phi}+\widehat{\phi}^{\dagger}\right), \quad \widehat{\theta}_{1}=-\frac{i}{2}\left(\widehat{\phi}-\phi^{\dagger}\right) .
$$

Provided that the condensate density $n_{0}$ and velocity $\vec{v}_{0}=$ $\hbar \nabla \theta_{0} / m$ vary on length scales much bigger than the healing length $\xi=\hbar / m c$ (the fundamental length scale of the condensate), the BdG equation reduces to the continuity and Euler equations for $\widehat{n}_{1}$ and $\widehat{\theta}_{1}$ and these can be combined to give a second-order differential equation for $\widehat{\theta}_{1}$ which is mathematically equivalent to a Klein-Gordon (KG) equation

$$
\square \widehat{\theta}_{1}=0,
$$

where $\square$ is the covariant KG operator from the acoustic metric

$$
d s^{2}=\frac{n_{0}}{m c}\left[-\left(c^{2}-\vec{v}_{0}^{2}\right) d t^{2}-2 \vec{v}_{0} d t d \vec{x}+d \vec{x}^{2}\right] .
$$

For a flow which presents a transition from a subsonic $\left(\left|v_{0}\right|<c\right)$ flow to a supersonic one $\left(\left|\vec{v}_{0}\right|>c\right.$ in some region) the metric (8) describes an acoustic black hole, with horizon located at the surface where $\left|v_{0}\right|=c$. The same analysis performed by Hawking in the gravitational case can be repeated step by step, leading to the prediction [17] that acoustic black holes will emit a thermal flux of phonons at the temperature

$$
T_{H}=\frac{\kappa}{2 \pi}
$$

where $\kappa=\left.(1 / 2 c)\left(d\left(c^{2}-\vec{v}^{2}\right) / d n\right)\right|_{\text {hor }}$, with $n$ being the normal to the horizon, is the horizon's surface gravity.

\section{The Model}

To simplify the mathematics involved in the process, we will consider a 1D configuration (in 1D one should more correctly speak of quasi-condensation $[18,19])$ in which $n_{0}$ and $v_{0}$ are constant and where the only nontrivial quantity is the speed of sound $c$. As explained in [20], this can be achieved by varying the coupling constant $g$ (and therefore $c$ ) and the external potential but keeping the sum $g n_{0}+V_{\text {ext }}$ constant. In this way, the plane-wave function $\Psi_{0}=\sqrt{n_{0}} e^{i k_{0} x-i w_{0} t}$, where $v_{0}=\hbar k_{0} / m$ is the condensate velocity and $\hbar w_{0}=$ $\hbar^{2} k_{0}^{2} / 2 m+V_{\text {ext }}+g n_{0}$, where $\hbar w_{0}$ is the chemical potential of the gas, is a solution of (4) everywhere. Note that such a stationary configuration is difficult to reach experimentally; nevertheless it gives results similar to those obtained by more realistic configurations [21].

The fluctuation operator $\widehat{\phi}$ is expanded in the usual form in terms of positive and negative norm modes as

$$
\widehat{\phi}(t, x)=\sum_{j}\left[\widehat{a}_{j} \phi_{j}(t, x)+\widehat{a}_{j}^{\dagger} \varphi_{j}^{*}(t, x)\right],
$$

where $\widehat{a}_{j}$ and $\widehat{a}_{j}^{\dagger}$ are quasi-particle's annihilation and creation operators. From (5) and its Hermitean conjugate, we see that the modes $\phi_{j}(t, x)$ and $\varphi_{j}(t, x)$ satisfy the coupled differential equations

$$
\begin{gathered}
{\left[i\left(\partial_{t}+v_{0} \partial_{x}\right)+\frac{\xi c}{2} \partial_{x}^{2}-\frac{c}{\xi}\right] \phi_{j}=\frac{c}{\xi} \varphi_{j}} \\
{\left[-i\left(\partial_{t}+v_{0} \partial_{x}\right)+\frac{\xi c}{2} \partial_{x}^{2}-\frac{c}{\xi}\right] \varphi_{j}=\frac{c}{\xi} \phi_{j}}
\end{gathered}
$$

The normalizations are fixed, via integration of the equal-time commutator obtained from (1), namely,

$$
\left[\widehat{\phi}(t, x), \widehat{\phi}^{\dagger}\left(t, x^{\prime}\right)\right]=\frac{1}{n_{0}} \delta\left(x-x^{\prime}\right),
$$

by

$$
\int d x\left[\phi_{j} \phi_{j^{\prime}}^{*}-\varphi_{j}^{*} \varphi_{j^{\prime}}\right]=\frac{\delta_{j j^{\prime}}}{n_{0}} .
$$

In order to get simple analytical expressions, in the following we will consider simple models with step-like discontinuities in the speed of sound $c$ and impose the appropriate boundary conditions for the modes that are solutions to (11). For more general profiles a numerical analysis can be performed; see, for example, [22-25]. 
3.1. Acoustic Black Holes and the Hawking Effect. A simple analytical model of an acoustic black hole [26] can be obtained by gluing two semi-infinite stationary and homogeneous 1D condensates, one subsonic $(x<0)$ and the other supersonic $(x>0)$, along a spatial discontinuity at $x=0$ (see [27], to which we refer for more detailed explanations throughout this paragraph, and the references therein): $c(x)=c_{l} \theta(-x)+c_{r} \theta(x)$. We take $v_{0}<0$; that is, the flow is from right to left, and $c_{l}<\left|v_{0}\right|<c_{r}$. We denote the modes solutions in each homogeneous region and corresponding to the fields $\phi$ and $\varphi$ as

$$
\phi_{\omega}=D(\omega) e^{-i \omega t+i k(\omega) x}, \quad \varphi_{\omega}=E(\omega) e^{-i \omega t+i k(\omega) x},
$$

so that (11) become

$$
\begin{aligned}
& {\left[\left(\omega-v_{0} k\right)-\frac{\xi c k^{2}}{2}-\frac{c}{\xi}\right] D(\omega)=\frac{c}{\xi} E(\omega),} \\
& {\left[-\left(\omega-v_{0} k\right)-\frac{\xi c k^{2}}{2}-\frac{c}{\xi}\right] E(\omega)=\frac{c}{\xi} D(\omega),}
\end{aligned}
$$

while the normalization condition (13) gives

$$
|D(\omega)|^{2}-|E(\omega)|^{2}=\frac{1}{2 \pi n_{0}}\left|\frac{d k}{d w}\right| .
$$

The combination of the two equations (15) gives the Bogoliubov dispersion relation for a one-dimensional Bose liquid flowing at constant velocity

$$
\left(\omega-v_{0} k\right)^{2}=c^{2}\left(k^{2}+\frac{\xi^{2} k^{4}}{4}\right)
$$

containing the positive and negative norm branches $w-v_{0} k=$ $\pm c \sqrt{k^{2}+\left(\xi^{2} k^{4} / 4\right)} \equiv \pm \Omega(k)$ which, for the subsonic and supersonic regions, are given, respectively, in Figures 1 and 2. Moreover, inserting the relation between $D$ and $E$ from (15) into (16) we find the mode normalizations

$$
\begin{aligned}
& D(\omega)=\frac{\omega-v k+\left(c \xi k^{2} / 2\right)}{\sqrt{4 \pi n_{0} c \xi k^{2}\left|(\omega-v k)(d k / d \omega)^{-1}\right|}}, \\
& E(\omega)=-\frac{\omega-v k-\left(c \xi k^{2} / 2\right)}{\sqrt{4 \pi n_{0} c \xi k^{2}\left|(\omega-v k)(d k / d \omega)^{-1}\right|}},
\end{aligned}
$$

where $k=k(\omega)$ are the roots of the quartic equation (17) at fixed $\omega$.

In the subsonic case (17) admits two real and two complex solutions. Regarding the real solutions, Figure 1 , we call $k_{v}(\sim$ $\left.\omega /\left(v_{0}-c\right)+O\left(\xi^{2}\right)\right)$ and $k_{u}\left(\sim \omega /\left(v_{0}+c\right)+O\left(\xi^{2}\right)\right)$ the ones corresponding to, respectively, negative and positive group velocity $v_{g}=d \omega / d k$ (the other two complex conjugated solutions correspond to, respectively, spatially decaying $k_{d}$ and growing $k_{g}$ modes). In the supersonic case (see Figure 2$)$ we see that, for the most interesting regime $\left(\omega<\omega_{\max } \sim 1 / \xi\right)$, there are now four real solutions, corresponding to four propagating modes: $k_{v}, k_{u}$ (present also in the hydrodynamical limit $\xi=0$ )

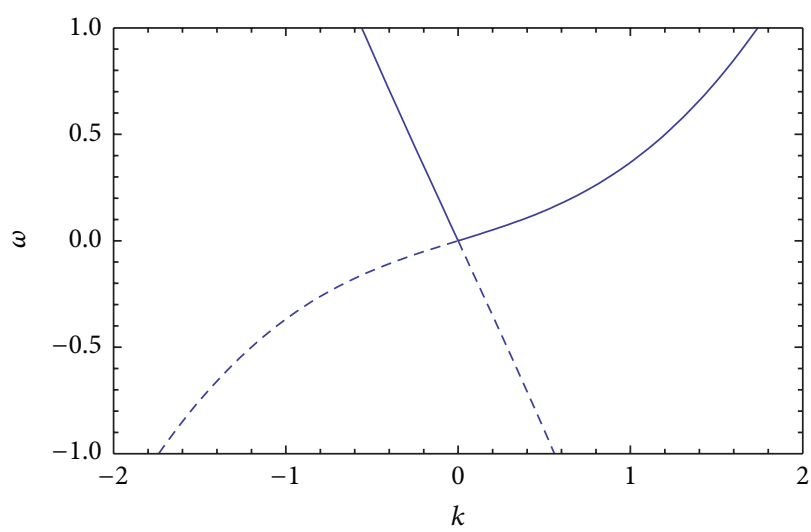

FIGURE 1: Dispersion relation in the subsonic region ( $\omega$ is given in units of the chemical potential and $k$ in units of the healing length).

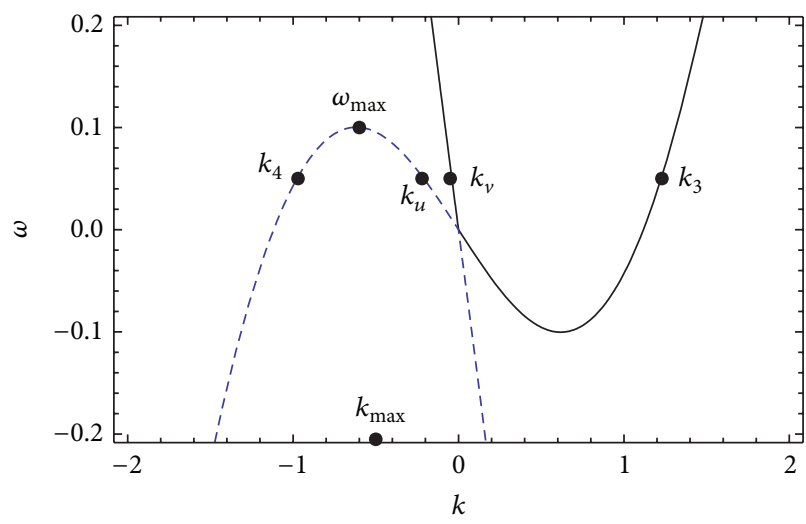

FIGURE 2: Dispersion relation in the supersonic region (again, $\omega$ is given in units of the chemical potential and $k$ in units of the healing length).

and $k_{3}, k_{4}(\sim 1 / \xi)$, two of which $\left(k_{u}\right.$ and $\left.k_{4}\right)$ belong to the negative norm branch.

To find modes evolution for all $x$ one needs to write down the general solutions for $\phi(\varphi)$ in the left supersonic $(l)$ and the right subsonic $(r)$ regions (we restrict to the most interesting case $\left.\omega<\omega_{\max }\right)$

$$
\begin{aligned}
\phi_{\omega}^{l}= & e^{-i \omega t} \\
& \times\left[D_{v}^{l} A_{v}^{l} e^{i k_{v}^{l} x}+D_{u}^{l} A_{u}^{l} e^{i k_{u}^{l} x}+D_{3}^{l} A_{3}^{l} e^{i k_{3}^{l} x}+D_{4}^{l} A_{4}^{l} e^{i k_{4}^{l} x}\right], \\
\phi_{\omega}^{r}= & e^{-i \omega t} \\
& \times\left[D_{v}^{r} A_{v}^{r} e^{i k_{v}^{r} x}+D_{u}^{r} A_{u}^{r} e^{i k_{u}^{r} x}+d_{\phi} A_{d}^{r} e^{i k_{d}^{3} x}+G_{\phi} A_{g}^{r} e^{i k_{g}^{r} x}\right]
\end{aligned}
$$

(the expansions for $\varphi$ are the same up to the replacement $D \rightarrow E$ ) and impose, from (11), the matching conditions

$$
[\phi]=0, \quad\left[\phi^{\prime}\right]=0, \quad[\varphi]=0, \quad\left[\varphi^{\prime}\right]=0,
$$


where [ ] indicates the variation across the jump at $x=0$, allowing us to write down the relations between left and right amplitudes $A$ through a scattering matrix $M_{\text {scatt }}$ in the form

$$
\left(\begin{array}{c}
A_{v}^{l} \\
A_{u}^{l} \\
A_{3}^{l} \\
A_{4}^{l}
\end{array}\right)=M_{\text {scatt }}\left(\begin{array}{c}
A_{v}^{r} \\
A_{u}^{r} \\
A_{d}^{r} \\
A_{g}^{r}
\end{array}\right) .
$$

This allows us to construct explicitly the decomposition of the field operator $\hat{\phi}$ in terms of the "in" and "out" bases. The "in" basis is constructed with $\phi^{\text {in }}$ modes propagating from the asymptotic regions $(x \rightarrow \pm \infty)$ towards the discontinuity $(x=0)$, while the "out" basis is constructed with modes $\phi^{\text {out }}$ propagating away from the discontinuity to $x= \pm \infty$. Looking at Figures 1 and 2, we see that unit amplitude modes defined on the left moving $k_{v}^{r}$ and right moving $k_{3}^{l}, k_{4}^{l}(\sim$ $1 / \xi)$ momenta define the ingoing scattering states, while unit amplitude modes defined on the right moving $k_{u}^{r}$ and left moving $k_{v}^{l}, k_{u}^{l}$ momenta define the outgoing scattering states. One can then write down the "in" decomposition in terms of the "in" scattering states

$$
\widehat{\phi}=\int_{0}^{\omega_{\max }} d \omega\left[\widehat{a}_{\omega}^{v, \text { in }} \phi_{\omega}^{v, \text { in }}+\widehat{a}_{\omega}^{3, \text { in }} \phi_{\omega}^{3, \text { in }}+\widehat{a}_{\omega}^{4, \text { in } \dagger} \phi_{\omega}^{4, \text { in }}+\text { h.c. }\right]
$$

or, equivalently, on the basis of the "out" scattering ones. Note that since $k_{4}^{l}$ belongs to the negative norm branch the corresponding "in" mode $\phi_{4, l}^{\text {in }}$ is multiplied by a creation operator $\widehat{a}_{\omega}^{4, \text { in }}$ (the same thing happens, in the "out" decomposition, for $\phi_{u, l}^{\text {out }}$ ). Using (21) one can construct the $3 \times 3$ S-matrix relating $\phi^{\text {in }}$ and $\phi^{\text {out }}$ modes

$$
\begin{aligned}
& \phi_{\omega}^{v \text {,in }}=S_{v l, v r} \phi_{\omega}^{v, \text { out }}+S_{u r, v r} \phi_{\omega}^{u r, \text { out }}+S_{u l, v l} \phi_{\omega}^{u l, \text { out }}, \\
& \phi_{\omega}^{3, \text { in }}=S_{v l, 3 l} \phi_{\omega}^{v, \text { out }}+S_{u r, 3 l} \phi_{\omega}^{u r, \text { out }}+S_{u l, 3 l} \phi_{\omega}^{u l, \text { out }}, \\
& \phi_{\omega}^{4, \text { in }}=S_{v l, 4 l} \phi_{\omega}^{v, \text { out }}+S_{u r, 4 l} \phi_{\omega}^{u r, \text { out }}+S_{u l, 4 l} \phi_{\omega}^{u l, \text { out }},
\end{aligned}
$$

which is not trivial since it mixes positive and negative norm modes. As a consequence, the Bogoliubov transformation between "in" and "out" creation and annihilation operators is also not trivial because it mixes creation and annihilation operators. This has the crucial consequence that the "in" and "out" Hilbert spaces are not unitary related; in particular the corresponding vacua are different; that is, $\mid 0$, in $\rangle \neq \mid 0$, out $\rangle$. The physical consequence is that if we prepare the system in the $\mid 0$, in $\rangle$ vacuum state, so that there are no incoming phonons at $t=-\infty$, we will have, at late times, outgoing quanta on both sides of the horizon: the vacuum has spontaneously emitted phonons, mainly in the $k_{u}^{r}$ channel (Hawking quanta) and $k_{u}^{l}$ (partners). The analytical calculations show that the number of emitted Hawking quanta [26]

$$
\left\langle 0, \text { in }\left|\widehat{a}_{\omega}^{u r, \text { out } \dagger} \widehat{a}_{\omega}^{u r, \text { out }}\right| 0, \text { in }\right\rangle=\left|S_{u r, 4 l}\right|^{2} \sim \frac{1}{\omega}
$$

and partners

$$
\left\langle 0, \text { in }\left|\widehat{a}_{\omega}^{u l, \text { out } \dagger} \widehat{a}_{\omega}^{u l, \text { out }}\right| 0, \text { in }\right\rangle=\left|S_{u l, 4 l}\right|^{2} \sim \frac{1}{\omega}
$$

follow an approximate (low-frequency) thermal $(1 / w)$ spectrum [21], the proportionality factor allowing the identification of a Hawking temperature $(\sim 1 / \xi)$ in this idealised setting. We can understand the mechanism by which Hawking radiation is emitted by looking, using (6), at the equal-time density-density correlator

$$
G^{(2)}\left(t ; x, x^{\prime}\right) \equiv \frac{1}{n_{0}^{2}} \lim _{t \rightarrow t^{\prime}}\left\langle 0, \text { in }\left|\widehat{n}^{1}(t, x), \widehat{n}^{1}\left(t^{\prime}, x^{\prime}\right)\right| 0, \text { in }\right\rangle,
$$

whose main contribution in the $x<0$ and $x^{\prime}>0$ sector comes from the $u l$-ur term

$$
\begin{aligned}
& \left.\frac{1}{n_{0}^{2}}\left\langle\widehat{n}_{1} \widehat{n}_{1}\right\rangle\right|_{\text {Hawking }} \\
& \sim \operatorname{Re} \int_{0}^{w_{\max }} d w S_{u l, 4 l} S_{u r, 4 l}^{*}\left(\phi_{u, l}^{w, \text { out }}+\varphi_{u, l}^{w, \text { out }}\right) \\
& \quad \times\left(\phi_{u, r}^{w, \text { out } *}+\varphi_{u, r}^{w, \text { out } *}\right) \\
& \sim \frac{\sin \left[\omega_{\max }\left(x^{\prime} /\left(v_{0}+c_{r}\right)-x /\left(v_{0}+c_{l}\right)\right)\right]}{x^{\prime} /\left(v_{0}+c_{r}\right)-x /\left(v_{0}+c_{l}\right)} .
\end{aligned}
$$

The existence of the peak at

$$
\frac{x^{\prime}}{v_{0}+c_{r}}=\frac{x}{v_{0}+c_{l}}
$$

was first pointed out in [14] in the hydrodynamical approximation using QFT in curved space techniques. The physical picture that emerges is that Hawking quanta and partners are continuously created in pairs from the horizon at each time $t$, propagate on opposite directions at speeds $v_{0}+c_{l}<0$ and $v_{0}+c_{r}>0$, and after time $\Delta t$ are located at $x$ and $x^{\prime}$ related as in (28). The existence of the Hawking peak was nicely confirmed by numerical "ab initio" simulations with more realistic configurations performed in [20]. How correlation measurements can reveal the quantum nature of Hawking radiation is discussed in [28-30].

3.2. Analog Dynamical Casimir Effect. A distinct type of paircreation takes place in time-dependent backgrounds, with one important example being quantum particle creation in cosmology. We will study the analogue of these phenomena in BECs with another simple model in which the speed of sound has a step-like discontinuity in time at $t=0$ separating two "initial" and "final" infinite homogeneous condensates: $c(t)=c_{\text {in }} \theta(-t)+c_{\text {fin }} \theta(t)$; see for more details [31, 32]. The modes solutions in the initial $(t<0)$ and final $(t>0)$ regions are now of the type

$$
\phi_{k}=D(k) e^{-i w(k) t+i k x}, \quad \varphi_{k}=E(k) e^{-i w(k) t+i k x} \text {, }
$$


for which (11) become

$$
\begin{aligned}
& {\left[-(\omega-v k)+\frac{c \xi k^{2}}{2}+\frac{c}{\xi}\right] D(k)=-\frac{c}{\xi} E(k),} \\
& {\left[(\omega-v k)+\frac{c \xi k^{2}}{2}+\frac{c}{\xi}\right] E(k)=-\frac{c}{\xi} D(k),}
\end{aligned}
$$

while the normalization condition (13) yields

$$
|D(k)|^{2}-|E(k)|^{2}=\frac{1}{2 \pi n_{0}}
$$

giving

$$
\begin{gathered}
D(k)=\frac{\omega-v k+\left(c \xi k^{2} / 2\right)}{\sqrt{4 \pi n_{0} c \xi k^{2}|(\omega-v k)|}}, \\
E(k)=-\frac{\omega-v k-\left(c \xi k^{2} / 2\right)}{\sqrt{4 \pi n_{0} c \xi k^{2}|(\omega-v k)|}} .
\end{gathered}
$$

Here, $\omega=\omega(k)$ corresponds to the two real solutions to (17), which is quadratic in $\omega$ at fixed $k$. These read

$$
\begin{aligned}
& \omega_{+}(k)=v k+\sqrt{c^{2} k^{2}+\frac{c^{2} k^{4} \xi^{2}}{4}} \equiv v k-\Omega(k), \\
& \omega_{-}(k)=v k-\sqrt{c^{2} k^{2}+\frac{c^{2} k^{4} \xi^{2}}{4}} \equiv v k-\Omega(k),
\end{aligned}
$$

where $\omega_{+}(k)$ corresponds to the positive norm branch and $\omega_{-}(k)$ corresponds to the negative norm one. To find modes evolution for all $t$ one first writes down the general solutions in the initial and final regions for $\phi(\varphi)$

$$
\begin{aligned}
\phi_{k}^{\mathrm{fin}(\mathrm{in})}= & e^{i k x} \\
& \times\left[D_{\text {fin(in) }}^{+}(k) A_{\mathrm{fin}(\mathrm{in})} e^{-i \omega_{+}^{\mathrm{fin}(\mathrm{in})}(k) t}\right. \\
& \left.+D_{\text {fin(in) }}^{-}(k) B_{\mathrm{fin}(\mathrm{in})} e^{-i \omega_{-}^{\mathrm{fin}(\mathrm{in})}(k) t}\right]
\end{aligned}
$$

(for $\varphi$ we have the same expansion with $D$ replaced by $E$ ) and impose the matching conditions, from (11),

$$
[\phi]=0, \quad[\varphi]=0,
$$

where [ ] now indicates the variation across the discontinuity at $t=0$. They allow the final amplitudes $A^{\text {fin }}$ to be related to the initial ones $A^{\text {in }}$ through the matrix $M_{\mathrm{Bog}}$ :

$$
\left(\begin{array}{l}
A_{\text {fin }} \\
B_{\text {fin }}
\end{array}\right)=M_{\text {bog }}\left(\begin{array}{c}
A_{\text {in }} \\
B_{\text {in }}
\end{array}\right),
$$

where

$$
M_{\text {bog }}=\frac{1}{2 \sqrt{\Omega_{\text {in }} \Omega_{\text {out }}}}\left(\begin{array}{ll}
\Omega_{\text {in }}+\Omega_{\text {fin }} & \Omega_{\text {fin }}-\Omega_{\text {in }} \\
\Omega_{\text {fin }}-\Omega_{\text {in }} & \Omega_{\text {in }}+\Omega_{\text {fin }}
\end{array}\right) .
$$

One can easily construct "in" and "fin" decompositions for the field $\widehat{\phi}$ by considering initial (final) unit amplitude positive norm modes $\left(A_{\text {in (fin) }}=1, B_{\text {in }}=0\right) \phi_{k}^{\text {in(fin) }}\left(\varphi_{k}^{\text {in(fin })}\right)$ modes for all $t$ using (36):

$$
\widehat{\phi}(t, x)^{\text {in (fin })}=\int_{-\infty}^{\infty} d k\left[\widehat{a}_{k}^{\text {in }(\text { fin })} \phi_{k}^{\text {in (fin })}+a_{k}^{\text {in }(\text { out }) \dagger} \varphi_{k}^{\text {in }(\text { fin }) *}\right] .
$$

The modes are related through a nontrivial Bogoliubov transformation mixing positive and negative norm modes

$$
\phi_{k}^{\text {in }}=\alpha_{k} \phi^{\text {fin }}+\beta_{k} \phi_{-k}^{\text {out } *}
$$

where

$$
\alpha_{k}=\frac{\Omega_{\mathrm{in}}+\Omega_{\mathrm{fin}}}{2 \sqrt{\Omega_{\mathrm{in}} \Omega_{\mathrm{fin}}}}, \quad \beta_{k}=\frac{\Omega_{\mathrm{fin}}-\Omega_{\mathrm{in}}}{2 \sqrt{\Omega_{\mathrm{in}} \Omega_{\mathrm{fin}}}},
$$

and, consequently, also the relation between "in" and "fin" annihilation and creation operators will mix annihilation and creation operators, implying again that the two decompositions are inequivalent and in particular the two vacuum states $\mid 0$, in $\rangle$ and $\mid 0$, fin $\rangle$ are different. The physical consequence is that the step-like discontinuity at $t=0$ will induce particle creation, the features of which can be understood by looking at the time-dependent terms of the one-time density-density correlator which in the hydrodynamical limit reads

$$
\begin{aligned}
& \left.G^{(2)}\left(t, x, x^{\prime}\right)\right|_{\text {dyncas }} \\
& \quad \sim \frac{1}{\left(2 c_{\text {fin }} t-\left(x-x^{\prime}\right)\right)^{2}}+\frac{1}{\left(2 c_{\text {fin }} t-\left(x^{\prime}-x\right)\right)^{2}} .
\end{aligned}
$$

At $t=0$ and everywhere in space correlated pairs of particles with opposite momentum are created out of the vacuum state, with velocities $v-c_{\text {fin }}$ (left moving) and $v+c_{\text {fin }}$ (right moving). At time $t$ such particles are separated by a distance

$$
\left|x-x^{\prime}\right|=2 c_{\mathrm{fin}} t
$$

which is indeed the correlation displayed in (41). This effect was recently observed in [33] by considering homogeneous condensates with trapping potential $V_{\text {ext }}$ rapidly varying in time, where correlation functions in velocity/momentum space were measured.

3.3. Amplification of the Hawking Signal in Density Correlators. It has been argued by Cornell [15] that a way to amplify the Hawking signal in density-density correlators is to reduce the interactions shortly before measuring the density correlations. Since $c=\sqrt{g n_{0} / m}$, reducing $g$ means that the speed of sound is also reduced. We will model this situation by matching our idealised acoustic black hole configuration of Section 3.1 with a final infinite homogeneous condensate characterised by a small sound velocity $c_{\text {fin }}\left(<c_{l}<\right.$ $c_{r}$ ); see Figure 3. To study this situation, in which a spatial step-like discontinuity in $c$ at $x=0$ is combined with a temporal step-like discontinuity at some $t=t_{0}$, we will use 
the tools introduced in the previous two subsections. We will calculate the density-density correlator in the "in" vacuum by expanding the density operator in the "in" decomposition

$$
\begin{array}{r}
\hat{n}^{1}(t, x) \\
\simeq n_{0} \int_{0}^{\omega_{\max }} d w\left[\widehat{a}_{\omega}^{v, \text { in }}\left(\phi_{v, r}^{\text {in }}+\varphi_{v, r}^{v, \text { in }}\right)+\widehat{a}_{\omega}^{3, \text { in }}\left(\phi_{3, l}^{\text {in }}+\varphi_{3, l}^{\text {in }}\right)\right. \\
\left.+\widehat{a}_{\omega}^{4, \text { in } \dagger}\left(\phi_{4, l}^{\text {in }}+\varphi_{4, l}^{\text {in }}\right)+h . c .\right]
\end{array}
$$

to get

$$
\begin{aligned}
\frac{1}{n_{0}^{2}}\left\langle 0, \text { in }\left|\widehat{n}_{1} \widehat{n}_{1}\right|\right. & 0, \text { in }\rangle \\
=\int_{0}^{w_{\max }} d w[ & \left(\phi_{v, r}^{\mathrm{in}}+\varphi_{v, r}^{v, \text { in }}\right)\left(\phi_{v, r}^{\mathrm{in} *}+\varphi_{v, r}^{v, \text { in } *}\right) \\
& +\left(\phi_{3, l}^{\mathrm{in}}+\varphi_{3, l}^{\mathrm{in}}\right)\left(\phi_{3, l}^{\mathrm{in} *}+\varphi_{3, l}^{\mathrm{in} *}\right) \\
& \left.+\left(\phi_{4, l}^{\mathrm{in} *}+\varphi_{4, l}^{\mathrm{in} *}\right)\left(\phi_{4, l}^{\mathrm{in}}+\varphi_{4, l}^{\mathrm{in}}\right)\right] .
\end{aligned}
$$

By expressing the "in" modes in terms of the "out" modes and in the absence of the temporal step-like discontinuity (say $t_{0} \rightarrow+\infty$ ) the Hawking signal is given by

$$
\begin{aligned}
\left.\frac{1}{n_{0}^{2}}\left\langle 0, \text { in }\left|\widehat{n}_{1} \widehat{n}_{1}\right| 0, \text { in }\right\rangle\right|_{\text {Hawking }} \\
\sim \operatorname{Re} \int_{0}^{w_{\max }} d w S_{u l, 4 l} S_{u r, 4 l}^{*}\left(\phi_{u, l}^{w, \text { out }}+\varphi_{u, l}^{w, \text { out }}\right) \\
\quad \times\left(\phi_{u, r}^{w, \text { out } *}+\varphi_{u, r}^{w, \text { out } *}\right) .
\end{aligned}
$$

In the presence of the temporal step-like discontinuity we need to evolve the relevant modes $\phi_{u, l}^{\text {out }}\left(\varphi_{u, l}^{\text {out }}\right)$ and $\phi_{u, r}^{\text {out }}\left(\varphi_{u, r}^{\text {out }}\right)$, at the same value of $w$, across the discontinuity at $t=t_{0}$. Going to the $k$ basis and considering $k, k^{\prime}$ small $\left(w \sim\left(v+c_{l}\right) k \sim\right.$ $\left.\left(v+c_{r}\right) k^{\prime}\right)$ we have

$$
\begin{aligned}
\phi_{u, l}^{k, \text { out }}+\varphi_{u, l}^{k, \text { out }} & \sim \sqrt{\frac{|k|}{c_{l}}} e^{-i\left(v+c_{l}\right) k t+i k x} \\
& \longrightarrow \sqrt{\frac{|k|}{c_{\text {fin }}}} e^{i k x}\left(\alpha e^{-i\left(v+c_{\text {fin }}\right) k t}+\beta e^{-i\left(v-c_{\text {fin }}\right) k t}\right),
\end{aligned}
$$

where $\alpha=\left(\left(c_{l}+c_{\text {fin }}\right) / 2 \sqrt{c_{l} c_{\text {fin }}}\right) e^{i\left(c_{\text {fin }}-c_{l}\right) k t_{0}}, \beta=\left(\left(c_{\text {fin }}-c_{l}\right) /\right.$ $\left.2 \sqrt{c_{l} c_{\text {fin }}}\right) e^{-i\left(c_{\text {fin }}+c_{l}\right) k t_{0}}$, and

$$
\begin{aligned}
\phi_{u, r}^{k^{\prime} \text { out }} & +\varphi_{u, r}^{k^{\prime} \text { out }} \\
& \sim \sqrt{\frac{k^{\prime}}{c_{r}}} e^{-i\left(v+c_{r}\right) k^{\prime} t+i k^{\prime} x^{\prime}} \\
& \longrightarrow \sqrt{\frac{k^{\prime}}{c_{\text {fin }}}} e^{i k^{\prime} x^{\prime}}\left(\alpha^{\prime} e^{-i\left(v+c_{\text {fin }}\right) k^{\prime} t}+\beta^{\prime} e^{-i\left(v-c_{\text {fin }}\right) k^{\prime} t}\right),
\end{aligned}
$$

FIGURE 3: Spacetime diagram sketch of a spatial step-like discontinuity (acoustic black hole-like) followed by a temporal one leading to a final homogeneous configuration.

with $\alpha^{\prime}=\left(\left(c_{r}+c_{\mathrm{fin}}\right) / 2 \sqrt{c_{r} c_{\mathrm{fin}}}\right) e^{i\left(c_{\mathrm{fin}}-c_{r}\right) k^{\prime} t_{0}}, \beta^{\prime}=\left(\left(c_{\mathrm{fin}}-\right.\right.$ $\left.\left.c_{r}\right) / 2 \sqrt{c_{r} c_{\text {fin }}}\right) e^{-i\left(c_{\text {fin }}+c_{r}\right) k^{\prime} t_{0}}$.

It is useful to rewrite (46) and (47) in terms of $w$ to compare with the standard result without the temporal steplike discontinuity

$$
\begin{aligned}
& \phi_{u, l}^{w, \text { out }}+\varphi_{u, l}^{w, \text { out }} \\
& \sim \sqrt{\frac{w}{c_{l}}} e^{-i w t+i\left(w x /\left(v+c_{l}\right)\right)} \longrightarrow \sqrt{\frac{w}{c_{\text {fin }}}} e^{i\left(w x /\left(v+c_{l}\right)\right)} \\
& \times\left(\alpha e^{-i\left(\left(v+c_{\text {fin }}\right) /\left(v+c_{l}\right)\right) w t}+\beta e^{-i\left(\left(v-c_{\text {fin }}\right) /\left(v+c_{l}\right)\right) w t}\right), \\
& \phi_{u, r}^{w, \text { out }}+\varphi_{u, r}^{w, \text { out }} \\
& \sim \sqrt{\frac{w}{c_{r}}} e^{-i w t+i\left(w x^{\prime} /\left(v+c_{r}\right)\right)} \longrightarrow \sqrt{\frac{w}{c_{\text {fin }}}} e^{i\left(w x^{\prime} /\left(v+c_{r}\right)\right)} \\
& \quad \times\left(\alpha^{\prime} e^{-i\left(\left(v+c_{\text {fin }}\right) /\left(v+c_{r}\right)\right) w t}+\beta^{\prime} e^{-i\left(\left(v-c_{\text {fin }}\right) /\left(v+c_{r}\right)\right) w t}\right) .
\end{aligned}
$$

The standard result is, from (45), a stationary peak at

$$
\frac{x}{v+c_{l}}-\frac{x^{\prime}}{v+c_{r}}=0
$$

weighted by the $w$-independent factor (see (24) and (25))

$$
\frac{w}{\sqrt{c_{l} c_{r}}} S_{u l, 4 l} S_{u r, 4 l}^{*}
$$

The effect of the temporal step-like discontinuity at $t=t_{0}$ (see (48)) is to modify this signal into a main signal located at

$$
\frac{x}{v+c_{l}}-\frac{x^{\prime}}{v+c_{r}}=\left(\frac{\left(v+c_{\mathrm{fin}}\right)}{v+c_{l}}-\frac{\left(v+c_{\mathrm{fin}}\right)}{v+c_{r}}\right)\left(t-t_{0}\right)
$$

with strength

$$
\frac{w \alpha \alpha^{\prime}}{c_{\mathrm{fin}}} S_{u l, 4 l} S_{u r, 4 l}^{*}
$$

and three smaller signals located at $x /\left(v+c_{l}\right)-x^{\prime} /\left(v+c_{r}\right)=$ $\left(\left(v+c_{\text {fin }}\right) /\left(v+c_{l}\right)-\left(v-c_{\text {fin }}\right) /\left(v+c_{r}\right)\right)\left(t-t_{0}\right), x /\left(v+c_{l}\right)-x^{\prime} /\left(v+c_{r}\right)=$ 
$\left(\left(v-c_{\text {fin }}\right) /\left(v+c_{l}\right)-\left(v+c_{\text {fin }}\right) /\left(v+c_{r}\right)\right)\left(t-t_{0}\right)$, and $x /\left(v+c_{l}\right)-x^{\prime} /(v+$ $\left.c_{r}\right)=\left(\left(v-c_{\text {fin }}\right) /\left(v+c_{l}\right)-\left(v-c_{\text {fin }}\right) /\left(v+c_{r}\right)\right)\left(t-t_{0}\right)$ with strengths given by (52) in which $\alpha \alpha$ ' is substituted, respectively, by $\alpha \beta^{\prime}$, $\alpha^{\prime} \beta$, and $\beta \beta^{\prime}$.

We see immediately that we loose the stationarity of the Hawking signal (49) and that the main signal is multiplied by

$$
\eta=\frac{\sqrt{c_{r} c_{l}}}{c_{\text {fin }}} \alpha \alpha^{\prime}=\frac{\left(c_{\text {fin }}+c_{l}\right)\left(c_{\text {fin }}+c_{r}\right)}{4 c_{\text {fin }}^{2}}
$$

with respect to the standard result. This results indeed in an amplification (i.e., the above term is $>1$ ) when $c_{\text {fin }}<c_{l}, c_{r}$. Being the Hawking peak expected to be of order $5 \times 10^{-3}$ for realistic experimental settings [20] (where $c_{r}=2 c_{l}$ was considered), we obtain an amplification factor $\eta=15 / 4$ for $c_{r}=2 c_{l}=4 c_{\text {fin }}$.

\section{Conclusions}

In this paper we have briefly reviewed the analysis of the analog Hawking effect and of the analog dynamical Casimir effect by considering simple analytical models of Bose-Einstein condensates in which the speed of sound has step-like discontinuities. We focussed on the study of the density-density correlators which show, in the former case, the existence of a characteristic stationary Hawking quantapartner peak located at (28) and, in the latter, that of a timedependent feature (42). Following a suggestion by Cornell, we combined these two analyses to construct a model in which the atoms' interactions are rapidly lowered (and so the speed of sound) before the correlations are measured. This results in an amplification of the main Hawking peak, now timedependent and located at (51), by the factor (53) that could be useful in the experimental search.

\section{Conflict of Interests}

The authors declare that there is no conflict of interests regarding the publication of this paper.

\section{Acknowledgment}

The authors thank I. Carusotto for useful discussions.

\section{References}

[1] S. W. Hawking, "Particle creation by black holes," Communications in Mathematical Physics, vol. 43, no. 3, pp. 199-220, 1975.

[2] L. J. Garay, J. R. Anglin, J. I. Cirac, and P. Zoller, "Sonic black holes in dilute Bose-Einstein condensates," Physical Review A, vol. 63, no. 2, Article ID 023611, 2001.

[3] L. J. Garay, J. R. Anglin, J. I. Cirac, and P. Zoller, "Sonic analog of gravitational black holes in Bose-Einstein condensates," Physical Review Letters, vol. 85, no. 22, pp. 4643-4647, 2000.

[4] O. Lahav, A. Itah, A. Blumkin et al., "Realization of a sonic black hole analog in a Bose-Einstein condensate," Physical Review Letters, vol. 105, no. 24, Article ID 240401, 2010.

[5] I. Shammass, S. Rinott, A. Berkovitz, R. Schley, and J. Steinhauer, "Phonon dispersion relation of an atomic Bose-Einstein condensate," Physical Review Letters, vol. 109, Article ID 195301, 2012.

[6] R. Schley, A. Berkovitz, S. Rinott, I. Shammass, A. Blumkin, and J. Steinhauer, "Planck distribution of phonons in a BoseEinstein condensate," Physical Review Letters, vol. 111, Article ID 055301, 2013.

[7] C. Barceló, S. Liberati, and M. Visser, “Towards the observation of Hawking radiation in Bose-Einstein condensates," International Journal of Modern Physics A, vol. 18, no. 21, pp. 3735-3745, 2003.

[8] C. Barceló, S. Liberati, and M. Visser, "Probing semiclassical analog gravity in Bose-Einstein condensates with widely tunable interactions," Physical Review A, vol. 68, Article ID 053613, 2003.

[9] S. Giovanazzi, C. Farrell, T. Kiss, and U. Leonhardt, "Conditions for one-dimensional supersonic flow of quantum gases," Physical Review A, vol. 70, no. 6, Article ID 063602, 2004.

[10] R. Schützhold, "Detection scheme for acoustic quantum radiation in Bose-Einstein condensates," Physical Review Letters, vol. 97, Article ID 190405, 2006.

[11] S. Wüster and C. M. Savage, "Limits to the analog Hawking temperature in a Bose-Einstein condensate," Physical Review A, vol. 76, Article ID 013608, 2007.

[12] Y. Kurita and T. Morinari, "Formation of a sonic horizon in isotropically expanding Bose-Einstein condensates," Physical Review A, vol. 76, no. 5, Article ID 053603, 2007.

[13] C. Barcelo, S. Liberati, and M. Visser, "Analogue gravity," Living Reviews in Relativity, vol. 8, article 12, 2005.

[14] R. Balbinot, A. Fabbri, S. Fagnocchi, A. Recati, and I. Carusotto, "Nonlocal density correlations as a signature of Hawking radiation from acoustic black holes," Physical Review A, vol. 78, no. 2, Article ID 021603, 2008.

[15] E. Cornell, "Towards the observation of Hawking radiation in condensed matter systems," Talk given at the workshop, Valencia, Spain, 2009, http://www.uv.es/workshopEHR.

[16] L. Pitaevskii and S. Stringari, Bose-Einstein Condensation, vol. 116 of International Series of Monographs on Physics, The Clarendon Press, Oxford, UK, 2003.

[17] W. G. Unruh, "Experimental black-hole evaporation?” Physical Review Letters, vol. 46, no. 21, pp. 1351-1353, 1981.

[18] Y. Castin, "Simple theoretical tools for low dimension Bose gases," Journal de Physique IV France, vol. 116, pp. 89-132, 2004.

[19] C. Mora and Y. Castin, "Extension of Bogoliubov theory to quasicondensates," Physical Review A, vol. 67, Article ID 053615, 2003.

[20] I. Carusotto, S. Fagnocchi, A. Recati, R. Balbino, and A. Fabbri, "Numerical observation of Hawking radiation from acoustic black holes in atomic Bose-Einstein condensates," New Journal of Physics, vol. 10, Article ID 103001, 2008.

[21] P.-É. Larré, A. Recati, I. Carusotto, and N. Pavloff, "Quantum fluctuations around black hole horizons in Bose-Einstein condensates," Physical Review A, vol. 85, no. 1, Article ID 013621, 2012.

[22] J. Macher and R. Parentani, "Black-hole radiation in BoseEinstein condensates," Physical Review A, vol. 80, no. 4, Article ID 043601, 2009.

[23] S. Finazzi and R. Parentani, "Spectral properties of acoustic black hole radiation: broadening the horizon," Physical Review D, vol. 83, no. 8, Article ID 084010, 2011.

[24] S. Finazzi and R. Parentani, "Hawking radiation in dispersive theories, the two regimes," Physical Review D, vol. 85, Article ID 124027, 2012. 
[25] S. Bar-Ad, R. Schilling, and V. Fleurov, "Nonlocality and fluctuations near the optical analog of a sonic horizon," Physical Review A, vol. 87, Article ID 013802, 2013.

[26] A. Recati, N. Pavloff, and I. Carusotto, "Bogoliubov theory of acoustic Hawking radiation in Bose-Einstein condensates," Physical Review A, vol. 80, no. 4, Article ID 043603, 2009.

[27] R. Balbinot, I. Carusotto, A. Fabbri, C. Mayoral, and A. Recati, "Understanding hawking radiation from simple models of atomic Bose-Einstein condensates," in Analogue Gravity Phenomenology, vol. 870 of Lecture Notes in Physics, pp. 181-219, 2013.

[28] K. V. Kheruntsyan, J.-C. Jaskula, P. Deuar et al., "Violation of the Cauchy-Schwarz inequality with matter waves," Physical Review Letters, vol. 108, Article ID 260401, 2012.

[29] J. R. M. de Nova, F. Sols, and I. Zapata, "Violation of CauchySchwarz inequalities by spontaneous Hawking radiation in resonant boson structures," http://arxiv.org/abs/1211.1761.

[30] S. Finazzi and I. Carusotto, "Entangled phonons in atomic BoseEinstein condensates," http://arxiv.org/abs/1309.3414.

[31] I. Carusotto, R. Balbinot, A. Fabbri, and A. Recati, "Density correlations and analog dynamical Casimir emission of Bogoliubov phonons in modulated atomic Bose-Einstein condensates," European Physical Journal D, vol. 56, no. 3, pp. 391-404, 2010.

[32] C. Mayoral, A. Fabbri, and M. Rinaldi, "Steplike discontinuities in Bose-Einstein condensates and Hawking radiation: dispersion effects," Physical Review D, vol. 83, Article ID 124047, 2011.

[33] J. C. Jaskula, G. B. Partridge, M. Bonneau et al., "Acoustic analog to the dynamical casimir effect in a Bose-Einstein condensate," Physical Review Letters, vol. 109, Article ID 220401, 2012. 

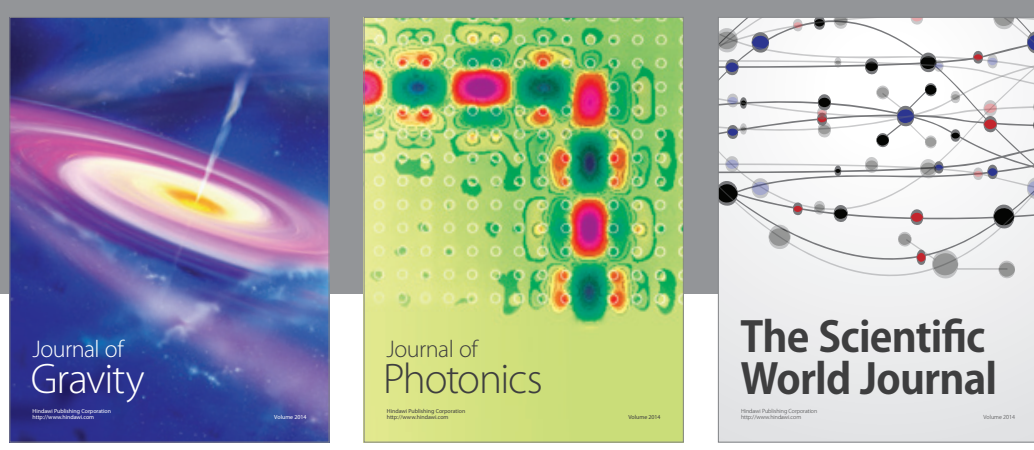

The Scientific World Journal
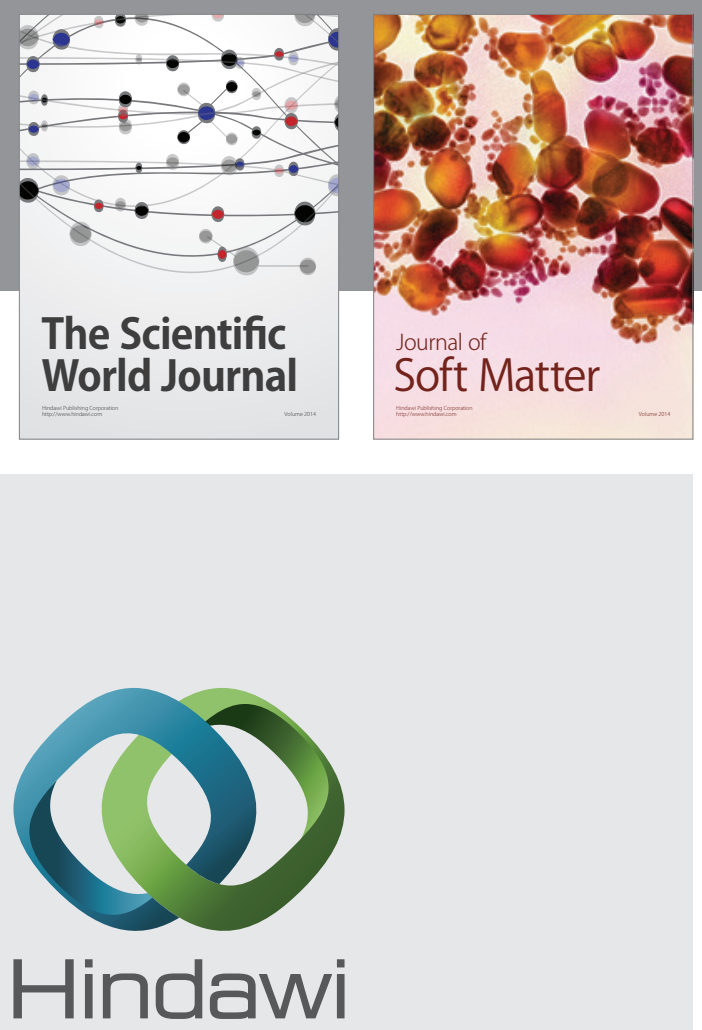

Submit your manuscripts at

http://www.hindawi.com

nternational Journal of

Statistical Mechanics
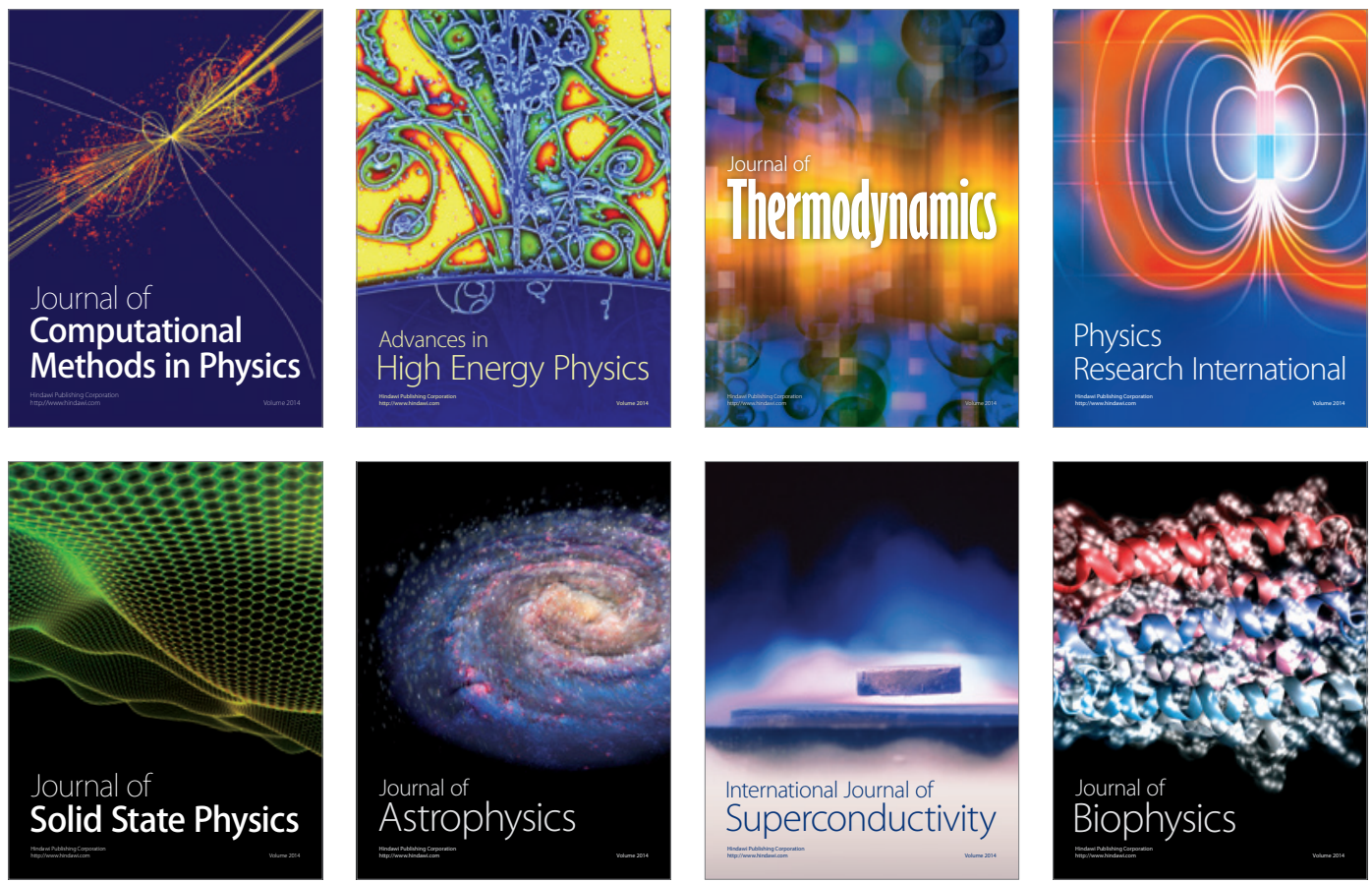
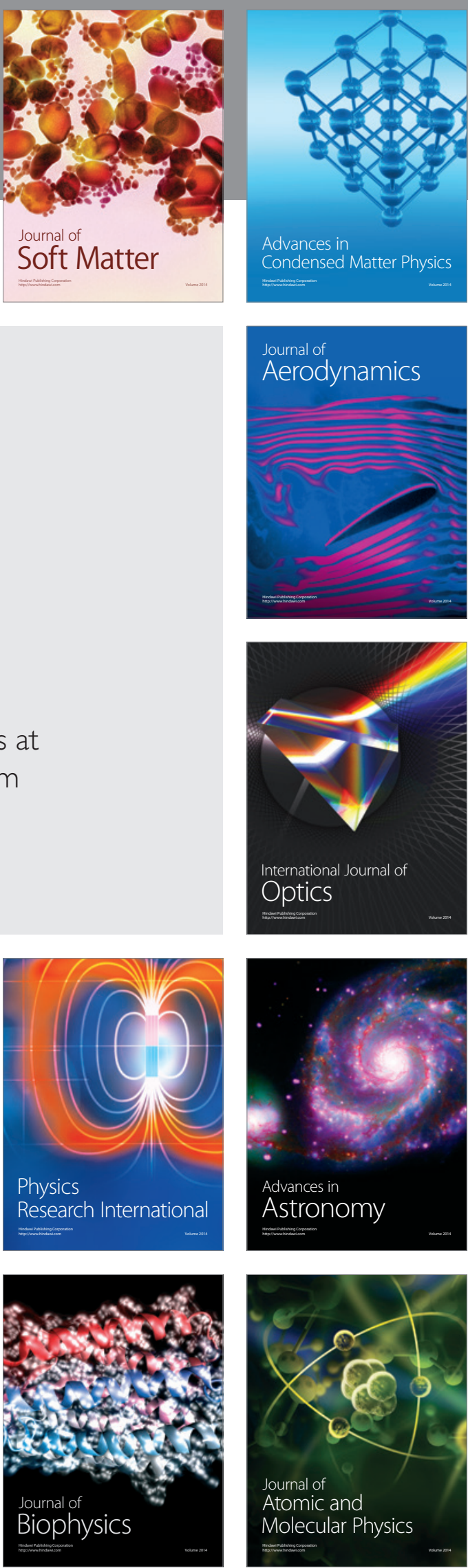